\title{
A NOTE ON COMMUTING ADDITIVE MAPS ON RANK K SYMMETRIC MATRICES*
}

\author{
WAI LEONG $\mathrm{CHOOI}^{\dagger}$ AND YEAN NEE TAN ${ }^{\dagger \ddagger}$
}

\begin{abstract}
Let $n \geqslant 2$ and $1<k \leqslant n$ be integers. Let $S_{n}(\mathbb{F})$ be the linear space of $n \times n$ symmetric matrices over a field $\mathbb{F}$ of characteristic not two. In this note, we prove that an additive map $\psi: S_{n}(\mathbb{F}) \rightarrow S_{n}(\mathbb{F})$ satisfies $\psi(A) A=A \psi(A)$ for all rank $k$ matrices $A \in S_{n}(\mathbb{F})$ if and only if there exists a scalar $\lambda \in \mathbb{F}$ and an additive map $\mu: S_{n}(\mathbb{F}) \rightarrow \mathbb{F}$ such that

$$
\psi(A)=\lambda A+\mu(A) I_{n}
$$

for all $A \in S_{n}(\mathbb{F})$, where $I_{n}$ is the identity matrix. Examples showing the indispensability of assumptions on the integer $k>1$ and the underlying field $\mathbb{F}$ of characteristic not two are included.
\end{abstract}

Key words. Commuting maps, Symmetric matrices, Ranks, Linear preserver problems, Functional identities.

AMS subject classifications. 15A03, 15A04, 15A86, 16R60.

1. Introduction. Motivated by mathematical interests, elegant results and applications, linear preserver problems on linear spaces of matrices has been an active research area in matrix theory since the last few decades. The formulation of linear preserver problems on matrices is natural, simple and of its applications. The study often enhances the understanding of the matrix functions, relations or identities under consideration, and also leads to interaction of matrix theory to other subjects. We refer the reader to the survey papers $[12,16]$ and the book [15] for history of linear preserver problems and its development.

Let $\mathcal{M}$ be a linear space of matrices. An additive map $\psi: \mathcal{M} \rightarrow \mathcal{M}$ is said to be commuting on a subset $\mathcal{H}$ of $\mathcal{M}$ provided that

$$
\psi(A) A=A \psi(A),
$$

for all $A \in \mathcal{H}$. In 2012, inspired by the theory of functional identities [2] and linear preserver problems, Franca [8] started to classify commuting additive maps $\psi: M_{n}(\mathbb{F}) \rightarrow M_{n}(\mathbb{F})$ on invertible (or singular) matrices, with $M_{n}(\mathbb{F})$ being the linear space of $n \times n$ matrices over a field $\mathbb{F}$. His proof was based on the structural result of Brešar [1, Theorem 3.2], and this work has advanced the study of functional identities to the set that is not closed under addition and multiplication. Later, many related results on commuting additive maps in this line of research have been published; see, for instance [4, 5, 6, 9, 10, 11, 13, 14, 18].

Let $n \geqslant 2$ be an integer and let $\mathbb{F}$ be a field of characteristic not two $(\operatorname{char} \mathbb{F} \neq 2)$. We denote by $S_{n}(\mathbb{F})$ the linear space of $n \times n$ symmetric matrices over $\mathbb{F}$. In this note, we address the question of describing the structure of commuting additive maps on symmetric matrices which are not closed under multiplication. More precisely, we characterize commuting additive maps $\psi: S_{n}(\mathbb{F}) \rightarrow S_{n}(\mathbb{F})$ on rank $k$ symmetric matrices, for some fixed integer $1<k \leqslant n$, and obtain the following result.

*Received by the editors on May 10, 2021. Accepted for publication on December 1, 2021. Handling Editor: Zejun Huang. Corresponding Author: Wai Leong Chooi.

${ }^{\dagger}$ Institute of Mathematical Sciences, Universiti Malaya, Kuala Lumpur, Malaysia (wlchooi@um.edu.my).

${ }^{\ddagger}$ Department of Pure and Applied Mathematics, School of Mathematical Sciences, Sunway University, Selangor, Malaysia (yeanneet@sunway.edu.my). 
Electronic Journal of Linear Algebra, ISSN 1081-3810

A publication of the International Linear Algebra Society

Volume 37, pp. 734-746, December 2021.

THEOREM 1. Let $n \geqslant 2$ be an integer and let $\mathbb{F}$ be a field with char $\mathbb{F} \neq 2$. Let $1<k \leqslant n$ be a fixed integer. Then, $\psi: S_{n}(\mathbb{F}) \rightarrow S_{n}(\mathbb{F})$ is a commuting additive map on rank $k$ symmetric matrices if and only if there exists a scalar $\lambda \in \mathbb{F}$ and an additive map $\mu: S_{n}(\mathbb{F}) \rightarrow \mathbb{F}$ such that

$$
\psi(A)=\lambda A+\mu(A) I_{n}
$$

for all $A \in S_{n}(\mathbb{F})$.

We will see some examples that the conditions $k>1$ and char $\mathbb{F} \neq 2$ in Theorem 1 are indispensable. The following result follows immediate from Theorem 1 and [3, Corollary 1.4].

COROLlary 2. Let $n \geqslant 3$ be an integer and let $\mathbb{F}$ be a field with char $\mathbb{F} \neq 2$. Let $k$ be a fixed integer such that $\frac{n}{2} \leqslant k \leqslant n$. Then $\psi: S_{n}(\mathbb{F}) \rightarrow S_{n}(\mathbb{F})$ is a map satisfying $\psi(A+B)=\psi(A)+\psi(B)$ and $\psi(A) A=A \psi(A)$ for all rank $k$ symmetric matrices $A, B \in S_{n}(\mathbb{F})$ if and only if there exists a scalar $\lambda \in \mathbb{F}$ and an additive $\operatorname{map} \mu: S_{n}(\mathbb{F}) \rightarrow \mathbb{F}$ such that

$$
\psi(A)=\lambda A+\mu(A) I_{n}
$$

for all $A \in S_{n}(\mathbb{F})$.

Derived from the work of Dolinar and Šemrl in [7], we deduce from Corollary 2 a characterization of maps on symmetric matrices satisfying Identity (1).

Corollary 3. Let $n \geqslant 3$ be an integer and let $\mathbb{F}$ be a field with char $\mathbb{F} \neq 2$. Then $\psi: S_{n}(\mathbb{F}) \rightarrow S_{n}(\mathbb{F})$ is a map satisfying

$$
\psi(A+B)(A+B)=(A+B)(\psi(A)+\psi(B))
$$

for all $A, B \in S_{n}(\mathbb{F})$ if and only if there exists a scalar $\lambda \in \mathbb{F}$ and an additive map $\mu: S_{n}(\mathbb{F}) \rightarrow \mathbb{F}$ such that

$$
\psi(A)=\lambda A+\mu(A) I_{n}
$$

for all $A \in S_{n}(\mathbb{F})$.

Given a pair of integers $1 \leqslant i, j \leqslant n$, we let $E_{i j} \in M_{n}(\mathbb{F})$ be the standard matrix unit whose $(i, j)$ th entry is one and zero elsewhere and denote $D_{i j}=E_{i j}+E_{j i}$ with $i \neq j$. Clearly, $D_{i j}=D_{j i}$ for any $i \neq j$. We now give examples to highlight the indispensability of $k>1$ and char $\mathbb{F} \neq 2$ in Theorem 1 .

ExAmple 4. Let $\mathbb{F}$ be the Galois field of two elements. Let $\psi: S_{2}(\mathbb{F}) \rightarrow S_{2}(\mathbb{F})$ be the additive map defined by:

$$
\psi(A)=\left(a_{11}+a_{12}+a_{22}\right)\left(\begin{array}{ll}
1 & 1 \\
1 & 1
\end{array}\right),
$$

for all $A=\left(a_{i j}\right) \in S_{2}(\mathbb{F})$. Let $A \in S_{2}(\mathbb{F})$ be of rank 2 . Then $A \in\left\{I_{2}, D_{12}, D_{12}+E_{11}, D_{12}+E_{22}\right\}$, and thus $\psi(A) A=A \psi(A)$. Hence, $\psi$ is a commuting additive map on rank two matrices.

ExAmPLE 5. Let $\mathbb{F}$ be the Galois field of two or three elements. Let $\psi: S_{2}(\mathbb{F}) \rightarrow S_{2}(\mathbb{F})$ be the additive map defined by:

$$
\psi(A)=\left(\begin{array}{cc}
0 & a_{12} \\
a_{12} & 0
\end{array}\right),
$$

for all $A=\left(a_{i j}\right) \in S_{2}(\mathbb{F})$. Let $A \in S_{2}(\mathbb{F})$ be of rank 1. Then

$$
A=\lambda\left(\begin{array}{ll}
a^{2} & a b \\
a b & b^{2}
\end{array}\right)
$$


for some scalars $\lambda, a, b \in \mathbb{F}$ with $\lambda \neq 0$ and $(a, b) \neq 0$. Notice that

$$
\psi(A) A=\lambda^{2} a b\left(\begin{array}{cc}
a b & b^{2} \\
a^{2} & a b
\end{array}\right) \quad \text { and } \quad A \psi(A)=\lambda^{2} a b\left(\begin{array}{cc}
a b & a^{2} \\
b^{2} & a b
\end{array}\right) .
$$

The result is clear when either $a=0$ or $b=0$. For the case $a \neq 0$ and $b \neq 0$, we see that $a^{2}=1=b^{2}$ when $|\mathbb{F}|=2,3$. It follows that $\psi$ is a commuting additive map on rank one matrices.

ExAMPLE 6 . Let $\mathbb{F}$ be a field with char $\mathbb{F} \neq 2$. Let $\psi: S_{3}(\mathbb{F}) \rightarrow S_{3}(\mathbb{F})$ be the additive map defined by:

$$
\psi(A)=\left(\begin{array}{ccc}
0 & -a_{23} & a_{22} \\
-a_{23} & 2 a_{13} & -a_{12} \\
a_{22} & -a_{12} & 0
\end{array}\right)
$$

for all $A=\left(a_{i j}\right) \in S_{3}(\mathbb{F})$. Let $A \in S_{3}(\mathbb{F})$ be of rank 1. Then

$$
A=\lambda\left(\begin{array}{lll}
a^{2} & a b & a c \\
a b & b^{2} & b c \\
a c & b c & c^{2}
\end{array}\right)
$$

for some scalars $\lambda, a, b, c \in \mathbb{F}$ with $\lambda \neq 0$ and $(a, b, c) \neq 0$. Note that

$$
\begin{gathered}
\psi(A) A=\left(\begin{array}{ccc}
0 & -\lambda b c & \lambda b^{2} \\
-\lambda b c & 2 \lambda a c & -\lambda a b \\
\lambda b^{2} & -\lambda a b & 0
\end{array}\right)\left(\lambda\left(\begin{array}{ccc}
a^{2} & a b & a c \\
a b & b^{2} & b c \\
a c & b c & c^{2}
\end{array}\right)\right)=0, \\
A \psi(A)=\lambda\left(\begin{array}{lll}
a^{2} & a b & a c \\
a b & b^{2} & b c \\
a c & b c & c^{2}
\end{array}\right)\left(\begin{array}{ccc}
0 & -\lambda b c & \lambda b^{2} \\
-\lambda b c & 2 \lambda a c & -\lambda a b \\
\lambda b^{2} & -\lambda a b & 0
\end{array}\right)=0 .
\end{gathered}
$$

Therefore, $\psi$ is a commuting additive map on rank one matrices.

2. Results. Let $n \geqslant 2$ be an integer and let $\mathbb{F}$ be a field. Let $\psi: S_{n}(\mathbb{F}) \rightarrow S_{n}(\mathbb{F})$ be an additive map. First note that for each pair of distinct integers $1 \leqslant p, q \leqslant n$, there exist additive maps $\phi_{i j}^{(p p)}: \mathbb{F} \rightarrow \mathbb{F}$, $1 \leqslant i \leqslant j \leqslant n$, and $\phi_{i j}^{(p q)}: \mathbb{F} \rightarrow \mathbb{F}, 1 \leqslant i \leqslant j \leqslant n$, such that

$$
\begin{aligned}
& \psi\left(a E_{p p}\right)=\sum_{i=1}^{n} \phi_{i i}^{(p p)}(a) E_{i i}+\sum_{1 \leqslant i<j \leqslant n} \phi_{i j}^{(p p)}(a) D_{i j}, \\
& \psi\left(a D_{p q}\right)=\sum_{i=1}^{n} \phi_{i i}^{(p q)}(a) E_{i i}+\sum_{1 \leqslant i<j \leqslant n} \phi_{i j}^{(p q)}(a) D_{i j},
\end{aligned}
$$

for all $a \in \mathbb{F}$. We start with a technical lemma to give a complete description of commuting additive maps $\psi: S_{n}(\mathbb{F}) \rightarrow S_{n}(\mathbb{F})$ on the subset $\mathcal{B}_{n}$ of $S_{n}(\mathbb{F})$ with char $\mathbb{F} \neq 2$, where

$$
\mathcal{B}_{n}=\left\{\begin{array}{cc}
\left\{\alpha E_{r r}+\beta D_{12}: \alpha, \beta \in \mathbb{F} \text { and } r=1,2\right\} & \text { if } n=2, \\
\left\{\alpha E_{r r}+\beta D_{s t}, \alpha D_{r s}+\alpha D_{s t}: \alpha, \beta \in \mathbb{F}, 1 \leqslant r, s, t \leqslant n \text { and } s \neq t\right\} & \text { if } n \geqslant 3 .
\end{array}\right.
$$

LEMmA 7. Let $n \geqslant 2$ be an integer and let $\mathbb{F}$ be a field with char $\mathbb{F} \neq 2$. Then, $\psi: S_{n}(\mathbb{F}) \rightarrow S_{n}(\mathbb{F})$ is a commuting additive map on $\mathcal{B}_{n}$ if and only if there exists a scalar $\lambda \in \mathbb{F}$ and an additive map $\mu: S_{n}(\mathbb{F}) \rightarrow \mathbb{F}$ such that

$$
\psi(A)=\lambda A+\mu(A) I_{n}
$$

for all $A \in S_{n}(\mathbb{F})$. 
Electronic Journal of Linear Algebra, ISSN 1081-3810

A publication of the International Linear Algebra Society

Volume 37, pp. 734-746, December 2021.

Proof. The sufficiency is obvious. We consider the necessity. Let $1 \leqslant s \leqslant n$ be an integer and $a \in \mathbb{F}$. Since $\psi\left(a E_{s s}\right)\left(a E_{s s}\right)=\left(a E_{s s}\right) \psi\left(a E_{s s}\right)$, it follows from (2) that

$$
\sum_{i=1}^{n} \phi_{i i}^{(s s)}(a) a\left(E_{i i} E_{s s}\right)+\sum_{1 \leqslant i<j \leqslant n} \phi_{i j}^{(s s)}(a) a\left(D_{i j} E_{s s}\right)=\sum_{i=1}^{n} a \phi_{i i}^{(s s)}(a)\left(E_{s s} E_{i i}\right)+\sum_{1 \leqslant i<j \leqslant n} a \phi_{i j}^{(s s)}(a)\left(E_{s s} D_{i j}\right) .
$$

We thus obtain

$$
\sum_{i=1}^{s-1}\left(\phi_{i s}^{(s s)}(a) a E_{i s}-a \phi_{i s}^{(s s)}(a) E_{s i}\right)+\sum_{j=s+1}^{n}\left(\phi_{s j}^{(s s)}(a) a E_{j s}-a \phi_{s j}^{(s s)}(a) E_{s j}\right)=0,
$$

for every $a \in \mathbb{F}$ and integer $1 \leqslant s \leqslant n$. Then for each integer $1 \leqslant p \leqslant n$,

$$
\begin{array}{ll}
\phi_{i p}^{(p p)}=0 & \text { for } i=1, \ldots, p-1, \\
\phi_{p j}^{(p p)}=0 & \text { for } j=p+1, \ldots, n .
\end{array}
$$

Let $1 \leqslant s<t \leqslant n$ be integers and $a \in \mathbb{F}$. We infer by $(3)$ and $\psi\left(a D_{s t}\right)\left(a D_{s t}\right)=\left(a D_{s t}\right) \psi\left(a D_{s t}\right)$ that

$$
\sum_{i=1}^{n} \phi_{i i}^{(s t)}(a) a\left(E_{i i} D_{s t}\right)+\sum_{1 \leqslant i<j \leqslant n} \phi_{i j}^{(s t)}(a) a\left(D_{i j} D_{s t}\right)=\sum_{i=1}^{n} a \phi_{i i}^{(s t)}(a)\left(D_{s t} E_{i i}\right)+\sum_{1 \leqslant i<j \leqslant n} a \phi_{i j}^{(s t)}(a)\left(D_{s t} D_{i j}\right) .
$$

Hence,

$$
\begin{aligned}
0=( & \left.\phi_{s s}^{(s t)}(a) a-a \phi_{t t}^{(s t)}(a)\right) E_{s t}+\left(\phi_{t t}^{(s t)}(a) a-a \phi_{s s}^{(s t)}(a)\right) E_{t s} \\
& +\sum_{i=1}^{s-1}\left(\phi_{i s}^{(s t)}(a) a E_{i t}-a \phi_{i s}^{(s t)}(a) E_{t i}\right)+\sum_{j=t+1}^{n}\left(\phi_{t j}^{(s t)}(a) a E_{j s}-a \phi_{t j}^{(s t)}(a) E_{s j}\right) \\
& +\sum_{i=1, i \neq s}^{t-1}\left(\phi_{i t}^{(s t)}(a) a E_{i s}-a \phi_{i t}^{(s t)}(a) E_{s i}\right)+\sum_{j=s+1, j \neq t}^{n}\left(\phi_{s j}^{(s t)}(a) a E_{j t}-a \phi_{s j}^{(s t)}(a) E_{t j}\right),
\end{aligned}
$$

for all $a \in \mathbb{F}$ and integers $1 \leqslant s<t \leqslant n$. Then for each pair of integers $1 \leqslant p<q \leqslant n$,

$$
\begin{gathered}
\phi_{q q}^{(p q)}=\phi_{p p}^{(p q)}, \\
\phi_{i p}^{(p q)}=0 \text { for } i=1, \ldots, p-1, \\
\phi_{q j}^{(p q)}=0 \text { for } j=q+1, \ldots, n, \\
\phi_{i q}^{(p q)}=0 \text { for } i=1, \ldots, q-1, \text { with } i \neq p, \\
\phi_{p j}^{(p q)}=0 \text { for } j=p+1, \ldots, n, \text { with } j \neq q .
\end{gathered}
$$

Let $1 \leqslant r, s, t \leqslant n$ be integers such that $s<t$ and let $a, b \in \mathbb{F}$. We consider $a E_{r r}+b D_{s t} \in \mathcal{B}_{n}$. By the additivity of $\psi$ and $\psi(S) S=S \psi(S)$ for $S \in\left\{a E_{r r}, b D_{s t}, a E_{r r}+b D_{s t}\right\}$, we obtain

$$
\psi\left(a E_{r r}\right) b D_{s t}+\psi\left(b D_{s t}\right) a E_{r r}=a E_{r r} \psi\left(b D_{s t}\right)+b D_{s t} \psi\left(a E_{r r}\right)
$$


From (2), we see that

$$
\begin{aligned}
\psi\left(a E_{r r}\right) b D_{s t}= & \phi_{s s}^{(r r)}(a) b E_{s t}+\phi_{t t}^{(r r)}(a) b E_{t s}+\sum_{i=1}^{t-1} \phi_{i t}^{(r r)}(a) b E_{i s}+\sum_{i=1}^{s-1} \phi_{i s}^{(r r)}(a) b E_{i t} \\
& +\sum_{j=t+1}^{n} \phi_{t j}^{(r r)}(a) b E_{j s}+\sum_{j=s+1}^{n} \phi_{s j}^{(r r)}(a) b E_{j t}, \\
b D_{s t} \psi\left(a E_{r r}\right)= & b \phi_{t t}^{(r r)}(a) E_{s t}+b \phi_{s s}^{(r r)}(a) E_{t s}+\sum_{i=1}^{t-1} b \phi_{i t}^{(r r)}(a) E_{s i}+\sum_{i=1}^{s-1} b \phi_{i s}^{(r r)}(a) E_{t i} \\
& +\sum_{j=t+1}^{n} b \phi_{t j}^{(r r)}(a) E_{s j}+\sum_{j=s+1}^{n} b \phi_{s j}^{(r r)}(a) E_{t j} .
\end{aligned}
$$

From (3), we have

$$
\begin{aligned}
& \psi\left(b D_{s t}\right) a E_{r r}=\phi_{r r}^{(s t)}(b) a E_{r r}+\sum_{i=1}^{r-1} \phi_{i r}^{(s t)}(b) a E_{i r}+\sum_{j=r+1}^{n} \phi_{r j}^{(s t)}(b) a E_{j r}, \\
& a E_{r r} \psi\left(b D_{s t}\right)=a \phi_{r r}^{(s t)}(b) E_{r r}+\sum_{i=1}^{r-1} a \phi_{i r}^{(s t)}(b) E_{r i}+\sum_{j=r+1}^{n} a \phi_{r j}^{(s t)}(b) E_{r j} .
\end{aligned}
$$

Substituting into (12), we obtain

$$
\begin{gathered}
\left(\phi_{s s}^{(r r)}(a) b-b \phi_{t t}^{(r r)}(a)\right) E_{s t}+\left(\phi_{t t}^{(r r)}(a) b-b \phi_{s s}^{(r r)}(a)\right) E_{t s}+\sum_{i=1, i \neq s}^{t-1}\left(\phi_{i t}^{(r r)}(a) b E_{i s}-b \phi_{i t}^{(r r)}(a) E_{s i}\right) \\
+\sum_{i=1}^{s-1}\left(\phi_{i s}^{(r r)}(a) b E_{i t}-b \phi_{i s}^{(r r)}(a) E_{t i}\right)+\sum_{j=t+1}^{n}\left(\phi_{t j}^{(r r)}(a) b E_{j s}-b \phi_{t j}^{(r r)}(a) E_{s j}\right) \\
+\sum_{j=s+1, j \neq t}^{n}\left(\phi_{s j}^{(r r)}(a) b E_{j t}-b \phi_{s j}^{(r r)}(a) E_{t j}\right)+\sum_{i=1}^{r-1}\left(\phi_{i r}^{(s t)}(b) a E_{i r}-a \phi_{i r}^{(s t)}(b) E_{r i}\right) \\
+\sum_{j=r+1}^{n}\left(\phi_{r j}^{(s t)}(b) a E_{j r}-a \phi_{r j}^{(s t)}(b) E_{r j}\right)=0,
\end{gathered}
$$

for all integers $1 \leqslant r, s, t \leqslant n$ with $s<t$, and scalars $a, b \in \mathbb{F}$. We distinguish five cases.

Case $1.1 \leqslant r=s<t \leqslant n$. Since $\phi_{i s}^{(s s)}=0$ for $i=1, \ldots, s-1$ by $(5), \phi_{s j}^{(s s)}=0$ for $j=s+1, \ldots, n$ by (6), $\phi_{i s}^{(s t)}=0$ for $i=1, \ldots, s-1$ by (8), and $\phi_{s j}^{(s t)}=0$ for $j=s+1, \ldots, t-1, t+1, \ldots, n$ by (11), it follows from (13) that

$$
\begin{aligned}
\left(\phi_{s s}^{(s s)}(a) b\right. & \left.-b \phi_{t t}^{(s s)}(a)-a \phi_{s t}^{(s t)}(b)\right) E_{s t}+\left(\phi_{t t}^{(s s)}(a) b+\phi_{s t}^{(s t)}(b) a-b \phi_{s s}^{(s s)}(a)\right) E_{t s} \\
& +\sum_{i=1, i \neq s}^{t-1} \phi_{i t}^{(s s)}(a) b E_{i s}-\sum_{i=1, i \neq s}^{t-1} b \phi_{i t}^{(s s)}(a) E_{s i}+\sum_{j=t+1}^{n} \phi_{t j}^{(s s)}(a) b E_{j s}-\sum_{j=t+1}^{n} b \phi_{t j}^{(s s)}(a) E_{s j}=0
\end{aligned}
$$

for all integers $1 \leqslant s<t \leqslant n$ and scalars $a, b \in \mathbb{F}$. Consequently, for each pair of integers $1 \leqslant p<q \leqslant n$,

$$
\begin{gathered}
\phi_{p p}^{(p p)}(a) b=b \phi_{q q}^{(p p)}(a)+a \phi_{p q}^{(p q)}(b) \text { for all } a, b \in \mathbb{F}, \\
\phi_{i q}^{(p p)}=0 \text { for } i=1, \ldots, q-1, \text { with } i \neq p, \\
\phi_{q j}^{(p p)}=0 \text { for } j=q+1, \ldots, n .
\end{gathered}
$$


Electronic Journal of Linear Algebra, ISSN 1081-3810

A publication of the International Linear Algebra Society

Volume 37, pp. 734-746, December 2021

By (14), we note that $\phi_{p q}^{(p q)}: \mathbb{F} \rightarrow \mathbb{F}$ is a linear map for every $1 \leqslant p<q \leqslant n$. It follows immediately that for each pair of integers $1 \leqslant p<q \leqslant n$, there exits a scalar $\tau_{p q} \in \mathbb{F}$ such that

$$
\begin{gathered}
\phi_{p q}^{(p q)}(a)=\tau_{p q} a \quad \text { for all } a \in \mathbb{F}, \\
\phi_{q q}^{(p p)}(a)=\phi_{p p}^{(p p)}(a)-\tau_{p q} a \text { for all } a \in \mathbb{F} .
\end{gathered}
$$

Case 2. $1 \leqslant s<t=r \leqslant n$. Since $\phi_{i t}^{(t t)}=0$ for $i=1, \ldots, t-1$ by $(5), \phi_{t j}^{(t t)}=0$ for $j=t+1, \ldots, n$ by $(6), \phi_{t j}^{(s t)}=0$ for $j=t+1, \ldots, n$ by $(9)$, and $\phi_{i t}^{(s t)}=0$ for $i=1, \ldots, s-1, s+1, \ldots, t-1$ by (10), it follows from (13) and (17) that

$$
\begin{aligned}
\left(\phi_{s s}^{(t t)}(a) b\right. & \left.+\tau_{s t} b a-b \phi_{t t}^{(t t)}(a)\right) E_{s t}+\left(\phi_{t t}^{(t t)}(a) b-b \phi_{s s}^{(t t)}(a)-a \tau_{s t} b\right) E_{t s} \\
& +\sum_{i=1}^{s-1} \phi_{i s}^{(t t)}(a) b E_{i t}-\sum_{i=1}^{s-1} b \phi_{i s}^{(t t)}(a) E_{t i}+\sum_{j=s+1, j \neq t}^{n} \phi_{s j}^{(t t)}(a) b E_{j t}-\sum_{j=s+1, j \neq t}^{n} b \phi_{s j}^{(t t)}(a) E_{t j}=0
\end{aligned}
$$

for all integers $1 \leqslant s<t \leqslant n$ and scalars $a, b \in \mathbb{F}$. We conclude that for each pair of integers $1 \leqslant p<q \leqslant n$,

$$
\begin{gathered}
\phi_{p p}^{(q q)}(a)=\phi_{q q}^{(q q)}(a)-\tau_{p q} a \text { for all } a \in \mathbb{F}, \\
\phi_{i p}^{(q q)}=0 \text { for } i=1, \ldots, p-1, \\
\phi_{p j}^{(q q)}=0 \text { for } j=p+1, \ldots, n, \text { with } j \neq q .
\end{gathered}
$$

From (18) and (19), we see that for each integer $1 \leqslant p \leqslant n$,

$$
\phi_{i i}^{(p p)}(a)=\left\{\begin{array}{ll}
\phi_{p p}^{(p p)}(a)-\tau_{i p} a & \text { if } 1 \leqslant i<p, \\
\phi_{p p}^{(p p)}(a)-\tau_{p i} a & \text { if } p<i \leqslant n
\end{array},\right.
$$

for all $a \in \mathbb{F}$. Moreover, from (5), (6), (15), (16), (20), and (21), for each integer $1 \leqslant p \leqslant n$,

$$
\phi_{i j}^{(p p)}=0 \text { for all integers } 1 \leqslant i<j \leqslant n .
$$

Consequently, by (2), (22), and (23), for each integer $1 \leqslant p \leqslant n$,

$$
\psi\left(a E_{p p}\right)=\phi_{p p}^{(p p)}(a) I_{n}-\sum_{i=1}^{p-1} \tau_{i p} a E_{i i}-\sum_{i=p+1}^{n} \tau_{p i} a E_{i i}
$$

for all $a \in \mathbb{F}$. When $n=2$, we infer by (24) that

$$
\psi\left(a E_{11}\right)=\left(\phi_{11}^{(11)}(a)-\tau_{12} a\right) I_{2}+\tau_{12} a E_{11} \quad \text { and } \quad \psi\left(a E_{22}\right)=\left(\phi_{22}^{(22)}(a)-\tau_{12} a\right) I_{2}+\tau_{12} a E_{22},
$$

for all $a \in \mathbb{F}$, and by (3), (7), and (17) that

$$
\psi\left(a D_{12}\right)=\phi_{11}^{(12)}(a) I_{2}+\tau_{12} a D_{12}
$$

for all $a \in \mathbb{F}$. Let $\mu: S_{2}(\mathbb{F}) \rightarrow \mathbb{F}$ be the additive map defined by:

$$
\mu(A)=\left(\phi_{11}^{(11)}\left(a_{11}\right)-\tau_{12} a_{11}\right)+\left(\phi_{22}^{(22)}\left(a_{22}\right)-\tau_{12} a_{22}\right)+\phi_{11}^{(12)}\left(a_{12}\right)
$$

for all $A=\left(a_{i j}\right) \in S_{2}(\mathbb{F})$. Hence, $\psi(A)=\lambda A+\mu(A) I_{2}$ for all $A \in S_{2}(\mathbb{F})$, where $\lambda=\tau_{12} \in \mathbb{F}$, as required. We now consider $n \geq 3$. 
Case 3. $1 \leqslant r<s<t \leqslant n$. Note that $\phi_{i j}^{(r r)}=0$ for all $1 \leqslant i<j \leqslant n$ by $(23)$, and $\phi_{r s}^{(s t)}=\phi_{r t}^{(s t)}=0$ by (8) and (10). Thus, Equation (13) can be simplified as follows:

$$
\begin{aligned}
\left(\phi_{s s}^{(r r)}(a) b\right. & \left.-b \phi_{t t}^{(r r)}(a)\right) E_{s t}+\left(\phi_{t t}^{(r r)}(a) b-b \phi_{s s}^{(r r)}(a)\right) E_{t s} \\
& +\sum_{i=1}^{r-1}\left(\phi_{i r}^{(s t)}(b) a E_{i r}-a \phi_{i r}^{(s t)}(b) E_{r i}\right)+\sum_{j=r+1, j \neq s, t}^{n}\left(\phi_{r j}^{(s t)}(b) a E_{j r}-a \phi_{r j}^{(s t)}(b) E_{r j}\right)=0,
\end{aligned}
$$

for all integers $1 \leqslant r<s<t \leqslant n$ and scalars $a, b \in \mathbb{F}$. Then, for each pair of integers $1 \leqslant p<q \leqslant n$,

$$
\begin{gathered}
\phi_{i i}^{(p p)}=\phi_{j j}^{(p p)} \text { for all } p<i<j \leqslant n, \\
\phi_{i j}^{(p q)}=0 \text { for all } 1 \leqslant i<j<p, \\
\phi_{i j}^{(p q)}=0 \quad \text { for all } 1 \leqslant i<p \text { and } i<j \leqslant n, \text { with } j \neq p, q .
\end{gathered}
$$

Case 4. $1 \leqslant s<r<t \leqslant n$. Likewise, since $\phi_{i j}^{(r r)}=0$ for all $1 \leqslant i<j \leqslant n$ by $(23)$, and $\phi_{r t}^{(s t)}=\phi_{s r}^{(s t)}=0$ by (10) and (11), it follows from (13) that

$$
\begin{aligned}
\left(\phi_{s s}^{(r r)}(a) b\right. & \left.-b \phi_{t t}^{(r r)}(a)\right) E_{s t}+\left(\phi_{t t}^{(r r)}(a) b-b \phi_{s s}^{(r r)}(a)\right) E_{t s} \\
& +\sum_{i=1, i \neq s}^{r-1}\left(\phi_{i r}^{(s t)}(b) a E_{i r}-a \phi_{i r}^{(s t)}(b) E_{r i}\right)+\sum_{j=r+1, j \neq t}^{n}\left(\phi_{r j}^{(s t)}(b) a E_{j r}-a \phi_{r j}^{(s t)}(b) E_{r j}\right)=0
\end{aligned}
$$

for all integers $1 \leqslant s<r<t \leqslant n$ and scalars $a, b \in \mathbb{F}$. Then for each pair of integers $1 \leqslant p<q \leqslant n$,

$$
\begin{gathered}
\phi_{i i}^{(p p)}=\phi_{j j}^{(p p)} \text { for all } 1 \leqslant i<p \text { and } p<j \leqslant n, \\
\phi_{i j}^{(p q)}=0 \text { for all } 1 \leqslant i<j \text { and } p<j<q, \text { with } i \neq p, \\
\phi_{i j}^{(p q)}=0 \text { for all } p<i<q \text { and } i<j \leqslant n, \text { with } j \neq q .
\end{gathered}
$$

Case 5. $1 \leqslant s<t<r \leqslant n$. Similarly, using $\phi_{i j}^{(r r)}=0$ for all $1 \leqslant i<j \leqslant n$ by $(23)$, and $\phi_{t r}^{(s t)}=\phi_{s r}^{(s t)}=0$ by (9) and (11), we thus obtain from (13) that

$$
\begin{aligned}
\left(\phi_{s s}^{(r r)}(a) b\right. & \left.-b \phi_{t t}^{(r r)}(a)\right) E_{s t}+\left(\phi_{t t}^{(r r)}(a) b-b \phi_{s s}^{(r r)}(a)\right) E_{t s} \\
& +\sum_{i=1, i \neq s, t}^{r-1}\left(\phi_{i r}^{(s t)}(b) a E_{i r}-a \phi_{i r}^{(s t)}(b) E_{r i}\right)+\sum_{j=r+1}^{n}\left(\phi_{r j}^{(s t)}(b) a E_{j r}-a \phi_{r j}^{(s t)}(b) E_{r j}\right)=0
\end{aligned}
$$

for all integers $1 \leqslant s<t<r \leqslant n$ and scalars $a, b \in \mathbb{F}$. Therefore, for each pair of integers $1 \leqslant p<q \leqslant n$,

$$
\begin{array}{cl} 
& \phi_{i i}^{(p p)}=\phi_{j j}^{(p p)} \text { for all } 1 \leqslant i<j<p, \\
\phi_{i j}^{(p q)}=0 \quad \text { for all } 1 \leqslant i<j \text { and } q<j \leqslant n, \text { with } i \neq p, q, & \phi_{i j}^{(p q)}=0 \text { for all } q<i<j \leqslant n .
\end{array}
$$

Then, we infer by (25), (28), and (30) that for each integer $1 \leqslant p \leqslant n$,

$$
\phi_{i i}^{(p p)}=\phi_{j j}^{(p p)} \quad \text { for all } i, j \in\{1, \ldots, n\} \backslash\{p\} .
$$

It follows from (22) and (32) that for each integer $1 \leqslant p \leqslant n$,

$$
\tau_{12}=\tau_{13}=\cdots=\tau_{1 n}
$$


Electronic Journal of Linear Algebra, ISSN 1081-3810

A publication of the International Linear Algebra Society

Volume 37, pp. 734-746, December 2021.

when $p=1$,

$$
\tau_{1 p}=\cdots=\tau_{p-1, p}=\tau_{p, p+1}=\cdots=\tau_{p n}
$$

when $1<p<n$, and

$$
\tau_{1 n}=\tau_{2 n}=\cdots=\tau_{n-1, n},
$$

when $p=n$. From this, we conclude that there exists a fixed scalar $\lambda \in \mathbb{F}$ such that

$$
\tau_{i j}=\lambda
$$

for all $1 \leqslant i<j \leqslant n$. From (24) and (33), for each integer $1 \leqslant p \leqslant n$,

$$
\psi\left(a E_{p p}\right)=\lambda a E_{p p}+\left(\phi_{p p}^{(p p)}(a)-\lambda a\right) I_{n}
$$

for all $a \in \mathbb{F}$. On the other hand, we infer by (8), (9), (10), (11), (26), (27), (29), and (31) that for each pair of integers $1 \leqslant p<q \leqslant n$,

$$
\phi_{i j}^{(p q)}=0 \text { for all } 1 \leqslant i<j \leqslant n \text {, with }(i, j) \neq(p, q) .
$$

From (3) and (35), we see that for each pair of integers $1 \leqslant p<q \leqslant n$,

$$
\psi\left(a D_{p q}\right)=\lambda a D_{p q}+\sum_{i=1}^{n} \phi_{i i}^{(p q)}(a) E_{i i}
$$

for all $a \in \mathbb{F}$.

We now proceed to claim that for each pair of integers $1 \leqslant p<q \leqslant n$,

$$
\phi_{i i}^{(p q)}=\phi_{p p}^{(p q)} \quad \text { for all } i=1, \ldots, n \text {. }
$$

Let $1 \leqslant r, s, t \leqslant n$ be distinct integers. We consider $1 \leqslant r<s<t \leqslant n$ and $a D_{r s}+a D_{s t} \in \mathcal{B}_{n}$ for $a \in \mathbb{F}$. By virtue of $\psi(S) S=S \psi(S)$ for $S \in\left\{a D_{r s}, a D_{s t}, a D_{r s}+a D_{s t}\right\}$, we have $\psi\left(a D_{r s}\right) a D_{s t}+\psi\left(a D_{s t}\right) a D_{r s}=$ $a D_{r s} \psi\left(a D_{s t}\right)+a D_{s t} \psi\left(a D_{r s}\right)$. It follows from (36) that

$$
\left(\phi_{r r}^{(s t)}(a) a-\phi_{s s}^{(s t)}(a) a\right)\left(E_{r s}-E_{s r}\right)+\left(\phi_{s s}^{(r s)}(a) a-\phi_{t t}^{(r s)}(a) a\right)\left(E_{s t}-E_{t s}\right)=0,
$$

for all integers $1 \leqslant r<s<t \leqslant n$ and scalars $a \in \mathbb{F}$. Then for each pair of integers $1<p<q \leqslant n$,

$$
\phi_{i i}^{(p q)}=\phi_{p p}^{(p q)} \quad \text { for } i=1, \ldots, p-1
$$

We now consider $1 \leqslant s<t<r \leqslant n$ and $a D_{s t}+a D_{t r} \in \mathcal{B}_{n}$ for $a \in \mathbb{F}$. Repeating the above argument, we obtain

$$
\left(\phi_{s s}^{(t r)}(a) a-\phi_{t t}^{(t r)}(a) a\right)\left(E_{s t}-E_{t s}\right)+\left(\phi_{t t}^{(s t)}(a) a-a \phi_{r r}^{(s t)}(a)\right)\left(E_{t r}-E_{r t}\right)=0,
$$

for all integers $1 \leqslant s<t<r \leqslant n$ and scalars $a \in \mathbb{F}$. Then, for each pair of integers $1 \leqslant p<q<n$,

$$
\phi_{i i}^{(p q)}=\phi_{q q}^{(p q)} \quad \text { for } i=q+1, \ldots, n
$$

Next, we consider $1 \leqslant s<r<t \leqslant n$ and $a D_{s r}+a D_{s t} \in \mathcal{B}_{n}$ for $a \in \mathbb{F}$. Repeating the above argument, we obtain

$$
\left(\phi_{r r}^{(s t)}(a) a-a \phi_{s s}^{(s t)}(a)\right)\left(E_{r s}-E_{s r}\right)+\left(\phi_{s s}^{(s r)}(a) b-b \phi_{t t}^{(s r)}(a)\right)\left(E_{s t}-E_{t s}\right)=0
$$


for all integers $1 \leqslant s<r<t \leqslant n$ and scalars $a \in \mathbb{F}$. Therefore, for each pair of integers $1 \leqslant p<q \leqslant n$,

$$
\phi_{i i}^{(p q)}=\phi_{p p}^{(p q)} \quad \text { for } i=p+1, \ldots, q-1 .
$$

From (38), (39), and (40), together with (7), the claim is proved. Consequently, we infer by (36) and (37) that for each pair of integers $1 \leqslant p<q \leqslant n$,

$$
\psi\left(a D_{p q}\right)=\lambda a D_{p q}+\phi_{p p}^{(p q)}(a) I_{n}
$$

for all $a \in \mathbb{F}$. Let $\mu: S_{n}(\mathbb{F}) \rightarrow \mathbb{F}$ be the additive map defined by:

$$
\mu(a E)=\left\{\begin{array}{cl}
\phi_{i i}^{(i i)}(a)-\lambda a & \text { if } E=E_{i i}, 1 \leqslant i \leqslant n, \\
\phi_{i i}^{(i j)}(a) & \text { if } E=D_{i j}, 1 \leqslant i<j \leqslant n,
\end{array}\right.
$$

for all $a \in \mathbb{F}$. By (34), (41), and (42) and the additivity of $\psi$ and $\mu$, we conclude that

$$
\begin{aligned}
\psi(A) & =\sum_{i=1}^{n} \psi\left(a_{i i} E_{i i}\right)+\sum_{1 \leqslant i<j \leqslant n} \psi\left(a_{i j} D_{i j}\right) \\
& =\sum_{i=1}^{n}\left(\lambda a_{i i} E_{i i}+\left(\phi_{i i}^{(i i)}\left(a_{i i}\right)-\lambda a_{i i}\right) I_{n}\right)+\sum_{1 \leqslant i<j \leqslant n}\left(\lambda a_{i j} D_{i j}+\phi_{i i}^{(i j)}\left(a_{i j}\right) I_{n}\right) \\
& =\lambda\left(\sum_{i=1}^{n} a_{i i} E_{i i}+\sum_{1 \leqslant i<j \leqslant n} a_{i j} D_{i j}\right)+\left(\sum_{i=1}^{n} \mu\left(a_{i i} E_{i i}\right)+\sum_{1 \leqslant i<j \leqslant n} \mu\left(a_{i j} D_{i j}\right)\right) I_{n} \\
& =\lambda A+\mu(A) I_{n},
\end{aligned}
$$

for all $A=\left(a_{i j}\right) \in S_{n}(\mathbb{F})$. This completes the proof.

As an immediate consequence of Lemma 7, we obtain the following corollary.

COROLlary 8. Let $n \geqslant 2$ be an integer and let $\mathbb{F}$ be a field with char $\mathbb{F} \neq 2$. Then $\psi: S_{n}(\mathbb{F}) \rightarrow S_{n}(\mathbb{F})$ is a commuting additive map on symmetric matrices if and only if there exists a scalar $\lambda \in \mathbb{F}$ and an additive $\operatorname{map} \mu: S_{n}(\mathbb{F}) \rightarrow \mathbb{F}$ such that

$$
\psi(A)=\lambda A+\mu(A) I_{n}
$$

for all $A \in S_{n}(\mathbb{F})$.

Let $A \in S_{n}(\mathbb{F})$ be a nonzero rank $k$ matrix. Then there exist nonzero scalars $\lambda_{1}, \ldots, \lambda_{k} \in \mathbb{F}$ and an invertible matrix $P \in M_{n}(\mathbb{F})$ such that

$$
A=P\left(\sum_{i=1}^{k} \lambda_{i} E_{i i}\right) P^{t}
$$

when char $\mathbb{F} \neq 2$; see, for example, [17, Corollary 1.33].

Lemma 9. Let $\mathbb{F}$ be a field with char $\mathbb{F} \neq 2$. Then each $A \in S_{2}(\mathbb{F})$ can be represented as a sum of three invertible matrices in $S_{2}(\mathbb{F})$ among which the sum of any two is invertible.

Proof. Let $A \in S_{2}(\mathbb{F})$. If $A=0$, then we set $X=Y=I_{2}$ and $Z=-2 I_{2}$. So $X, Y, Z \in S_{2}(\mathbb{F})$ are invertible such that $A=X+Y+X$ and the sum of any two is also invertible. If $A$ is of rank one, then we 
Electronic Journal of Linear Algebra, ISSN 1081-3810

A publication of the International Linear Algebra Society

Volume 37, pp. 734-746, December 2021.

assume without loss of generality that $A=E_{11}$ by (43). We set $X=D_{12}, Y=E_{11}+D_{12}$ and $Z=-2 D_{12}$. Then $X, Y, Z \in S_{2}(\mathbb{F})$ are invertible such that $A=X+Y+Z$ and the sum of any two is invertible. If $A$ is of rank two, then by (43) we may assume that $A=E_{11}+\lambda E_{22}$ for some nonzero $\lambda \in \mathbb{F}$. Take

$$
X=E_{11}-E_{22}-D_{12}, \quad Y=E_{22}-D_{12} \quad \text { and } \quad Z=E_{22}+2 D_{12},
$$

when $\lambda=1$, and

$$
X=E_{11}+(\lambda-1) D_{12}, \quad Y=\lambda E_{22}-\lambda D_{12} \quad \text { and } \quad Z=D_{12},
$$

when $\lambda \neq 1$. Then $X, Y, Z \in S_{2}(\mathbb{F})$ are invertible such that $A=X+Y+Z$ and the sum of any two is invertible. We are done.

LEMMA 10. Let $n$ and $k$ be integers such that $n \geqslant 3$ and $1<k \leqslant n$. Let $\mathbb{F}$ be a field with char $\mathbb{F} \neq 2$. If $A \in S_{n}(\mathbb{F})$ is of rank at most three, then $A$ can be represented as a sum of three rank $k$ matrices in $S_{n}(\mathbb{F})$ among which the sum of any two is of rank $k$.

Proof. Let $A \in S_{n}(\mathbb{F})$ be of rank at most three. If $A=0$, then $A=X+Y+Z$, where

$$
X=\sum_{i=1}^{k} E_{i i}, \quad Y=X \quad \text { and } \quad Z=-2 X .
$$

It is immediate that $X, Y$, and $Z$ are rank $k$ symmetric matrices such that the sum of any two is of rank $k$.

We consider $A$ is of rank one. By (43), we assume without loss of generality that $A=E_{11}$. Let

$$
X=D_{12}+\sum_{i=3}^{k} E_{i i}, \quad Y=E_{11}+X \quad \text { and } \quad Z=-2 X,
$$

where it is understood that $\sum_{i=3}^{k} E_{i i}=0$ when $k=2$. Then $A=X+Y+Z$ is the sum of three rank $k$ matrices $X, Y, Z \in S_{n}(\mathbb{F})$ among which the sum of any two is of rank $k$.

We next consider $A$ is of rank two. Invoking (43), we may assume $A=E_{11}+\lambda E_{22}$ for some nonzero $\lambda \in \mathbb{F}$. Two cases are considered below:

Case A1. $\lambda=1$. Set

$$
\begin{gathered}
X=E_{11}-E_{22}+D_{12}+\sum_{i=3}^{k} E_{i i} \\
Y=D_{12}+\sum_{i=2}^{k} E_{i i} \quad \text { and } \quad Z=E_{22}-2 D_{12}-\sum_{i=3}^{k} 2 E_{i i} .
\end{gathered}
$$

Then $A=X+Y+Z$ is the sum of three rank $k$ matrices $X, Y, Z \in S_{n}(\mathbb{F})$ among which the sum of any two is of rank $k$.

Case A2. $\lambda \neq 1$. Let

$$
\begin{gathered}
X=E_{11}+(1-\lambda) D_{12}+\sum_{i=3}^{k}(1-\lambda) E_{i i} \\
Y=\lambda D_{12}+\sum_{i=2}^{k} \lambda E_{i i} \quad \text { and } \quad Z=-D_{12}-\sum_{i=3}^{k} E_{i i}
\end{gathered}
$$


Then $A=X+Y+Z$ is the sum of three rank $k$ matrices $X, Y, Z \in S_{n}(\mathbb{F})$ among which the sum of any two is of rank $k$.

We now consider $A$ is of rank three. By virtue of (43), we may assume $A=E_{11}+\lambda E_{22}+\mu E_{33}$ for some nonzero $\lambda, \mu \in \mathbb{F}$. If $k=2$, then we take

$$
X=2 E_{11}+\mu E_{33}, \quad Y=\lambda E_{22}-\mu E_{33} \quad \text { and } \quad Z=-E_{11}+\mu E_{33} .
$$

Clearly, $A=X+Y+Z$ is the sum of three rank two matrices $X, Y, Z \in S_{n}(\mathbb{F})$ among which the sum of any two is of rank two. Suppose that $k \geqslant 3$. We distinguish three cases.

Case B1. $\lambda=\mu=1$. Set

$$
\begin{gathered}
X=E_{11}-E_{22}-E_{33}+D_{12}+D_{23}+\sum_{i=4}^{k} E_{i i} \\
Y=D_{12}+D_{23}+\sum_{i=2}^{k} E_{i i} \quad \text { and } \quad Z=E_{22}+E_{33}-2 D_{12}-2 D_{23}-\sum_{i=4}^{k} 2 E_{i i} .
\end{gathered}
$$

Then $A=X+Y+Z$ is the sum of three rank $k$ matrices $X, Y, Z \in S_{n}(\mathbb{F})$ among which the sum of any two is of rank $k$.

Case B2. $\lambda=1$ and $\mu \neq 1$. Let

$$
\begin{gathered}
X=E_{11}-D_{12}-D_{23}-\mu E_{33}+\sum_{i=4}^{k} E_{i i}, \\
Y=E_{22}+2 D_{12}+2 D_{23}+\mu E_{33}+\sum_{i=4}^{k} E_{i i} \quad \text { and } \quad Z=-D_{12}-D_{23}+\mu E_{33}-\sum_{i=4}^{k} 2 E_{i i} .
\end{gathered}
$$

We see that $A=X+Y+Z$ is the sum of three rank $k$ matrices $X, Y, Z \in S_{n}(\mathbb{F})$ among which the sum of any two is of rank $k$.

Case B3. $\lambda, \mu \neq 1$ and $\lambda \neq \mu$. Taking

$$
\begin{gathered}
X=E_{11}-D_{12}-D_{23}-\mu E_{33}+\sum_{i=4}^{k} E_{i i}, \\
Y=\lambda E_{22}+2 D_{12}+2 D_{23}+\mu E_{33}+\sum_{i=4}^{k} E_{i i} \quad \text { and } \quad Z=-D_{12}-D_{23}+\mu E_{33}-\sum_{i=4}^{k} 2 E_{i i},
\end{gathered}
$$

we see that $A=X+Y+Z$ is the sum of three rank $k$ matrices $X, Y, Z \in S_{n}(\mathbb{F})$ among which the sum of any two is of rank $k$. This completes our proof.

We are now ready to prove the main theorem.

Proof of Theorem 1. The sufficiency is trivial. We consider the necessity. Let $A \in \mathcal{B}_{n}$. In view of (4), we note that $A$ is of rank at most three when $n \geqslant 3$. It follows from Lemmas 9 and 10 that $A=X+Y+Z$ for some rank $k$ matrices $X, Y, Z \in S_{n}(\mathbb{F})$ such that $X+Y, Y+Z$ and $X+Z$ are of rank $k$. Since $\psi$ is a commuting additive map on rank $k$ symmetric matrices, it follows that $\psi(S)(S)=(S) \psi(S)$ for each $S \in\{X, Y, X+Y\}$. This yields

$$
\psi(X) Y+\psi(Y) X=Y \psi(X)+X \psi(Y)
$$


Electronic Journal of Linear Algebra, ISSN 1081-3810

A publication of the International Linear Algebra Society

Volume 37, pp. 734-746, December 2021

Likewise, we obtain $\psi(Y) Z+\psi(Z) Y=Z \psi(Y)+Y \psi(Z)$ and $\psi(X) Z+\psi(Z) X=Z \psi(X)+X \psi(Z)$. By the additivity of $\psi$, we obtain

$$
\begin{aligned}
\psi(A) A & =\psi(X+Y+Z)(X+Y+Z) \\
& =\psi(X) X+\psi(Y) Y+\psi(Z) Z+(\psi(X) Y+\psi(Y) X)+(\psi(Y) Z+\psi(Z) Y)+(\psi(X) Z+\psi(Z) X) \\
& =X \psi(X)+Y \psi(Y)+Z \psi(Z)+(Y \psi(X)+X \psi(Y))+(Z \psi(Y)+Y \psi(Z))+(Z \psi(X)+X \psi(Z)) \\
& =(X+Y+Z) \psi(X+Y+Z) \\
& =A \psi(A)
\end{aligned}
$$

Hence, $\psi$ is commuting on $\mathcal{B}_{n}$. The result follows immediately from Lemma 7 .

LEMma 11. [3, Corollary 1.4] Let $n \geqslant 3$ be an integer and let $\mathbb{F}$ be a field with char $\mathbb{F} \neq 2$. If $k$ is a fixed integer such that $\frac{n}{2} \leqslant k \leqslant n$, then a map $\psi: S_{n}(\mathbb{F}) \rightarrow S_{n}(\mathbb{F})$ satisfies $\psi(A+B)=\psi(A)+\psi(B)$ for all rank $k$ matrices $A, B \in S_{n}(\mathbb{F})$ if and only if $\psi$ is additive.

Proof of Corollary 2. The sufficiency is clear. For the necessity, it follows from the hypothesis and Lemma 11 that $\psi$ is a commuting additive map on rank $k$ symmetric matrices. The result follows immediately from Theorem 1.

Proof of Corollary 3. We consider the sufficiency. Note that

$$
\begin{aligned}
\psi(A+B)(A+B) & =\left(\lambda(A+B)+\mu(A+B) I_{n}\right)(A+B) \\
& =\lambda A^{2}+\lambda B^{2}+\lambda A B+\lambda B A+\mu(A) A+\mu(B) B+\mu(A) B+\mu(B) A \\
& =A \lambda A+A \mu(A)+A \lambda B+A \mu(B)+B \lambda A+B \mu(A)+B \lambda B+B \mu(B) \\
& =(A+B)(\psi(A)+\psi(B)),
\end{aligned}
$$

for all $A, B \in S_{n}(\mathbb{F})$. For the necessity, we first show that $\psi(0)=0$. Note that $\psi\left(I_{n}\right)=\psi\left(I_{n}+0\right)\left(I_{n}+\right.$ $0)=\left(I_{n}+0\right)\left(\psi\left(I_{n}\right)+\psi(0)\right)=\psi\left(I_{n}\right)+\psi(0)$ yields $\psi(0)=0$. Then, $\psi(A) A=\psi(A+0)(A+0)=(A+$ $0)(\psi(A)+\psi(0))=A \psi(A)$ for all $A \in S_{n}(\mathbb{F})$, and thus $\psi$ is a commuting map on $S_{n}(\mathbb{F})$. We now show that $\psi(2 A)=2 \psi(A)$ and $\psi(-A)=-\psi(A)$ for all invertible $A \in S_{n}(\mathbb{F})$. Let $A \in S_{n}(\mathbb{F})$ be invertible. Note that $2 A \psi(2 A)=\psi(2 A)(2 A)=\psi(A+A)(A+A)=(A+A)(\psi(A)+\psi(A))=2 A(2 \psi(A))$ yields $\psi(2 A)=2 \psi(A)$ because $2 A$ is invertible. Next, $A \psi(A)=\psi(A) A=\psi(-A+2 A)(-A+2 A)=(-A+2 A)(\psi(-A)+\psi(2 A))=$ $A(\psi(-A)+2 \psi(A))$ leads to $\psi(-A)=-\psi(A)$. Finally, we show that $\psi(A+B)=\psi(A)+\psi(B)$ for all invertible $A, B \in S_{n}(\mathbb{F})$. Let $A, B \in S_{n}(\mathbb{F})$ be invertible. Then

$$
\begin{aligned}
A \psi(A) & =\psi(A) A \\
& =\psi((A+B)+(-B))((A+B)+(-B)) \\
& =((A+B)+(-B))(\psi(A+B)+\psi(-B)) \\
& =A(\psi(A+B)-\psi(B)) .
\end{aligned}
$$

It follows that $\psi(A+B)=\psi(A)+\psi(B)$ for all invertible matrices $A, B \in S_{n}(\mathbb{F})$. The result follows immediately from Corollary 2.

Acknowledgment. The authors express their gratitude to anonymous referee for careful reading of the paper and for useful suggestions. The authors' research was supported by FRGS Research Grant Scheme: FRGS/ 1/2018/STG06/UM/02/9 (FP082-2018A). 


\section{REFERENCES}

[1] M. Brešar. Centralizing mappings and derivations in prime rings. J. Algebra, 156:385-394, 1993.

[2] M. Brešar, M.A. Chebotar, and W.S. Martindale. Functional Identities, 3rd edi. Birkhäuser Verlag, Basel, 2007.

[3] W.L. Chooi and K.H. Kwa. Additive maps of rank $r$ tensors and symmetric tensors. Linear Multilinear Algebra, 67:1269$1293,2019$.

[4] W.L. Chooi, K.H. Kwa, and L.Y. Tan. Commuting maps on invertible triangular matrices over $\mathbb{F}_{2}$. Linear Algebra Appl., 583:77-101, 2019.

[5] W.L. Chooi, K.H. Kwa, and L.Y. Tan. Commuting maps on rank $k$ triangular matrices. Linear Multilinear Algebra, 68:1021-1030, 2020 .

[6] W.L. Chooi and J.Y. Wong. Commuting additive maps on tensor products of matrices. Linear Multilinear Algebra, 2021, doi:10.1080/03081087.2021.1920876.

[7] G. Dolinar and P. Šemrl. Determinant preserving maps on matrix algebras. Linear Algebra Appl., 348:189-192, 2002.

[8] W. Franca. Commuting maps on some subsets of matrices that are not closed under addition. Linear Algebra Appl., 437:388-391, 2012.

[9] W. Franca. Commuting maps on rank- $k$ matrices. Linear Algebra Appl., 438:2813-2815, 2013.

[10] W. Franca. Weakly commuting maps on the set of rank-1 matrices. Linear Multilinear Algebra, 65:475-495, 2017.

[11] W. Franca and N. Louza. Commuting maps on rank-1 matrices over noncommutative division rings. Comm. Algebra, 45:4696-4706, 2017.

[12] C.K. Li and S. Pierce. Linear preserver problems. Amer. Math. Monthly, 108:591-605, 2001.

[13] C-K. Liu. Centralizing maps on invertible or singular matrices over division rings. Linear Algebra Appl., 440:318-324, 2014.

[14] C-K. Liu and J-J. Yang. Power commuting additive maps on invertible or singular matrices. Linear Algebra Appl., 530:127$149,2017$.

[15] L. Molnár. Selected Preserver Problems on Algebraic Structures of Linear Operators and on Function Spaces. Lecture Notes in Mathematics. Vol. 1895, Springer-Verlag, 2007.

[16] S. Pierce, M.H. Lim, R. Loewy, et al., A survey of linear preserver problems. Linear Multilinear Algebra, 33:1-129, 1992.

[17] Z.X. Wan. Geometry of Matrices. World Scientific, Singapore, 1996.

[18] X. Xu and X. Yi, Commuting maps on rank- $k$ matrices. Electron. J. Linear Algebra, 27:735-741, 2014. 